\title{
Comparative Analysis of Impact of Radiation from Mobile Phone on Human Brain Activity:GSM 2G vs GSM 3G
}

\author{
Ms.Supriya Goel ${ }^{1}$, Dr. Manoj Duhan ${ }^{2}$ and Dr. Geeta Singh $^{3}$ \\ ${ }^{1,2}$ E.C.E Department, ${ }^{3}$ B.M.E Department \\ 1,2,3 D.C.R.U.S.T,Murthal \\ goel.supriya03@gmail.com, \\ Duhan_manoj@rediffmail.com,Singhgeeta_23@rediffmail.com
}

\begin{abstract}
The fast and unrelenting spread of wireless telecommunication devices has changed the landscape of the telecommunication world, as we know it, principally in current years. There had been a great concern for possible health from exposure to the RF fields produced by these wireless technologies. Aim of this paper is to examine the impact of mobile phone radiations generated by $2 G$ and $3 G$ GSM mobile phone technology on the human brain. activity and compares it with idle condition via experimental works. The experiment has been conducted in a laboratory using different human volunteers. The period of operation is 10 minutes as the talking time on the phone. Electroencephalogram is used to monitor and capture the brain signals of various subjects during the experimental analysis for an interval of 10 minutes with two different communication technologies (GSM2G, GSM3G,IDLE condition). The signals obtained under three different conditioned are analyzed with digital signal processing techniques such as Autocorrelation, Cross Correlation and Power Spectral Density analyses. The result shows that use of GSM $2 G$ mobile phone has the larger effect on brain activity followed by GSM $3 G$ phone which is followed by idle condition.
\end{abstract}

Keywords: $2 G, 3 G, E D G E, E E G, G P P, G S M, S A R$

\section{Introduction}

The Wireless communication is experiencing a dynamic growth in the global scale and the cell phones are becoming a vital device in the global modern society as they allow people to maintain constant and uninterrupted communication without hampering their freedom of movement. Mobile phones use electromagnetic spectrum in the microwave range. The WHO have classified mobile phone radiation on the IARC scale into Group 2B possibly carcinogenic. That means that there "could be some risk" of carcinogenicity, so additional research into the long-term, heavy use of mobile phones needs to be conducted. This signal produces electromagnetic radiation in the form of thermal waves that are transferred to the body which causes health problems like high blood pressure and other symptoms such as hot ears, burning skin, headaches and fatigue, particularly at the place near the ear cranium region where they are known to affect the neurons. [1]People using cell phones are prone to high blood pressure and other symptoms such as hot ears, burning skin, headaches and fatigue. There have been various studies into the connection between mobile phones and memory loss. The amount of radio frequency energy a cell phone emits depends on the technology on which phone works, the distance between the phone's antenna and the person talking on the phone, the extent of the conversation and the distance of user from cell phone towers.

The mobile phone has changed rapidly over time and continue to put up, which means that human exposures also change over time. There had been increasing apprehension 
about the possible undesirable health effects resulting, exposure to radiofrequency radiations, radiated from mobile communication devices.

So, there is a great demand to study in the laboratory about the effects that can lead to health impairment from mobile phone radiations [2]. This renewal information can be used as a basis for new exposure limits that take into account of thermal and non-thermal effects of microwave radiations from cellular phones and base stations.

\section{Mobile Phone Radiation}

The electromagnetic environment consists of natural radiation and man-made intentionally or as by-products of the use of electrical and electromagnetic fields that are produced either electronics devices. The everyday use of these devices and systems emitting radio frequency is continuously increasing electromagnetic fields in the environment. Cellular mobile communication networks cause on average low levels of general electromagnetic fields in areas accessible to the public. Electromagnetic radiation can be classified into ionizing and non-ionizing radiation. [3]Ionizing radiation is the radiation with high energy which is able to remove tight bonds between electrons and atoms resulting in issue damage while non-ionizing radiation is the radiation that has enough energy to vibrate the atoms and molecule but do not remove the electrons in the molecule [20]. This radiation mainly occurs at low frequency range. Mobile phone is designed with low power transceiver to transmit voice and data to base station is located at few kilometers [5]. So, most of the effects studied are mainly due to non-ionizing radiations. Radio

frequency used to communicate by mobile Phone has the ability to penetrate through semi-solid substances like living tissue to a distance proportional to its power density [4]. It can cause thermal effect [18-19]. Thermal effects are the temperature rise in the body because of energy absorption from electromagnetic radiation. Thermal radiations are also induced when mobile phone is used to make a call or receive a call for a long period of time. Thermal radiation penetrates into biological tissues[1]. The established biophysical mechanisms underlying the interaction of RF radiation with cells, tissues and entire bodies include ionization potential, induced charge and specific Absorption Rate (SAR) where it is defined as the rate of RF power absorbed per unit mass by any part of the body [17]. SAR values are dependent on the separation distance of the body and the mobile phones. The nearer the distances of the radiation source to the human head, the higher the SAR values [22]. Mobile phone communication takes place by the transfer of signals via electromagnetic wave through radio frequency and microwave signal. So, there has been growing concern about the possible adverse health effects resulting from exposure to these radiofrequency radiations (RFR) emitted by the mobile phones.

\section{Action Potential}

The action potential is the electrical activity developed in the muscle or nerves during any activity. Action potentials occurred when different ions across the neuron membrane. For normal human being the amplitude of the Action potential ranges between $-58 \mathrm{mV}$ and $12 \mathrm{mV}$. Stimuli first opens the sodium channels because on outside there are many more sodium ions, and inside the neuron is negative as relative to the outside, sodium ions moves into the neuron. Remember, sodium has a positive charge, so neurons become more positive and then become depolarized. It takes longer for potassium channels to open. When they open, potassium moves out of the cell, reverse the depolarization. Also at this time, sodium channels tend to close. This causes the action potential to go back toward $-68 \mathrm{mV}$. The action potential actually goes past $-68 \mathrm{mV}$ because the potassium channels stay open a long. progressively, the ion concentrations go back to resting levels and the cell returns to $-68 \mathrm{mV}[4]$. 


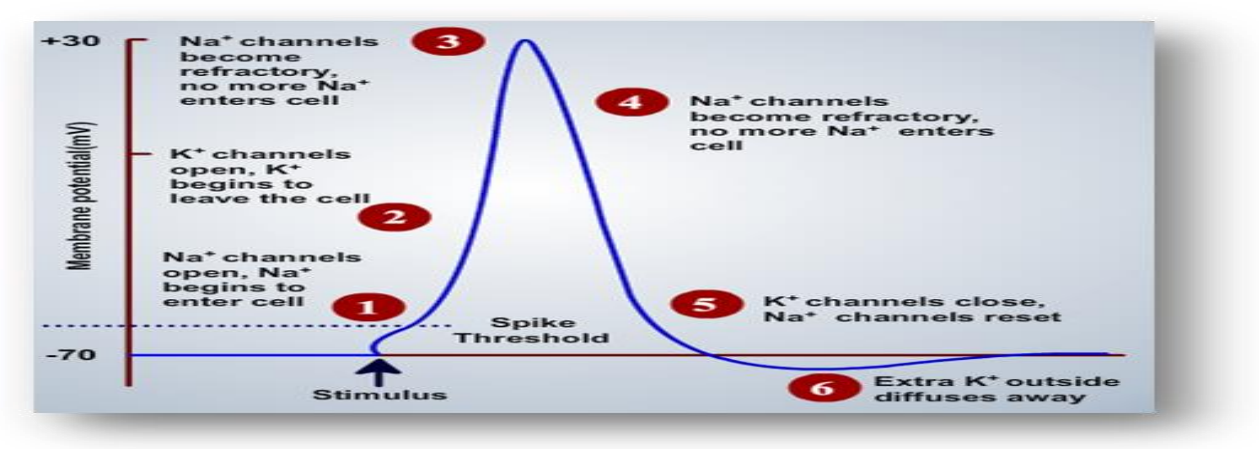

Figure 1. Action Potentional[6]

\section{GSM}

GSM abbreviated as Global System for Mobile Communications is the world's most popular standard for mobile telephone systems. GSM is the only wireless technology that provides worldwide reach, flawless global roaming with such a strong and versatile technology standard. GSM is the startup in the digital wireless communication next to the existing analog cellular communication technology. The GSM technology which was introduced in 1991 has got a great respnse from the subscribers internationally [3], due to which the number of subscribers has been increased to more than 200 million. This is the technology provide a highly protected and lucid communication. GSM uses two technologies FDMA abbreviated as Frequency Division Multiple Access and TDMA abbreviated as Time Division Multiple Access that allows multiple subscribers to make calls at the same time. GSM was the first telecommunication benchmark to commence the concept of a cell, where a cell takes the job to cover a small area. The GSM technology has taken the advantage of spectrum utilization which is divided into different bands depending on the location. The existing bands in Asia and Europe are GSM 900 and GSM 1800 [DCS] and the bands in the USA and Canada are GSM 850 and GSM 1900 respectively. The accessible bandwidth for $2 \mathrm{G}$ communication technologies is $200 \mathrm{kHz}$ per user and the air interface data rate is 270kbps.[7] GSM was designed predominantly for voice telephony, but after that a range of bearer services was introduced allowing circuit-switched data connections at upto $9600 \mathrm{bits} / \mathrm{s}$

\section{3. $2 \mathrm{G}$ and $3 \mathrm{G}$}

The first generation $1 \mathrm{G}$ cellular network that exists in 1980 transfers the data which includes only voice in analog wave but it has a disadvantage because there is no encryption and the the sound quality is very poor and its speed is $10 \mathrm{kbps}$. $2 \mathrm{G}$ is the improved technology which introduces the concept of digital modulation, which converts the voice into digital code. Being digital, they overcome many of the limitations of $1 \mathrm{G}$, such as it emits the radio power from handsets making life more healthy, and it increases privacy. The second generation $(2 \mathrm{G})$ is made up of the first digital mobile systems, mainly GSM (the technology found in more than $85 \%$ of the terminals used globally, with networks in practically all countries). [4] The digital signals consume less battery power, so they helps in saving the battery of mobile phones. Even though $2 \mathrm{G}$ phones are less expensive, they don't offer fast web browsing and file transfers as $3 \mathrm{G}$ phone can. 3G is used to represent the 3rd generation of mobile telecommunications technology. The $3 \mathrm{G}$ allows data, pictures and videos to be uploaded and downloaded at extremely fast rates. The data transmission speed on a $3 \mathrm{G}$ network ranges between $384 \mathrm{Kbps}$ to 2 Mbps[11]. An essential belief of the 3G GSM Initiative is an operation that promotes a common approach to the use of terminology. In all public and international communications the GSM will adopt the term "3GSM" to describe next generation GSM 
network and service contributions. 3GSM represents third generation services delivered on an evolved core GSM network. 3GSM services are delivered at a technological level on third generation developed by 3GPP, [6] which make use of Wideband-CDMA and, in some markets, EDGE air interfaces [5]. This said, the 3GSM standard is significantly more powerful than any other wireless technology and that competitive advantage need to be promoted cooperatively. No other standard offers such flexible combination of voice and data and capacity delivered by 3GSM. No other standard is as open as 3GSM.

\section{EEG}

An electroencephalogram (EEG) is a check that measures and records the electrical activity of the brain or we can say that it is a test used to detect abnormalities related to electrical movement of the brain. This procedure track and records brain wave pattern. Little metal discs with electrodes are placed on the scalp, and then send these accumulated signals to the computer for recording the results. Normally 1 electrical activity in the brain makes an identifiable pattern. With the help of EEG, doctors look for abnormal patterns that indicate seizure and any other problems.

An Electroencephalogram (EEG) has done to:

- For Diagnosing epilepsy and to see the type of seizures occurring.

- It checks the problems associated with unconsciousness.

- It helps to find out a person's chance of recovery after a change in consciousness[10].

- It finds out if a person who is in a coma is having dead brain.

- It study sleep disorders which are known as narcolepsy.

- It watches the brain activity while a person is receiving general anesthesia during brain surgery.

- It helps in finding out if a person has a physical problem (problems in the brain, spinal cord, or nervous system) or a mental health problem.

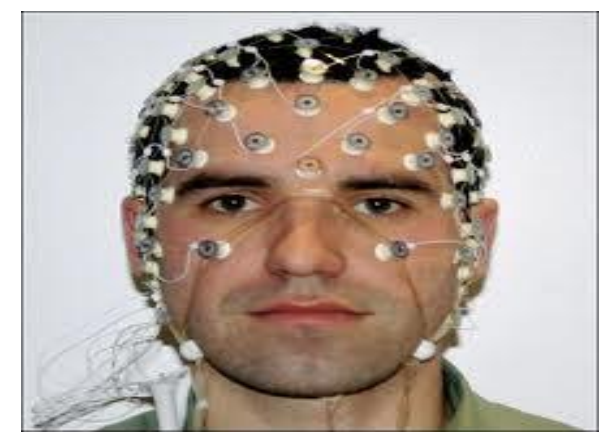

Figure 2. Placement of EEG on a Person

The Brain patterns which we obtained from EEG form wave shapes that are commonly sinusoidal are divided into five basic groups:

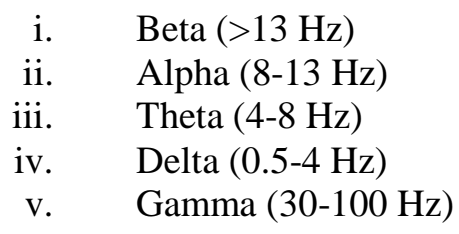

Delta is the waves having a frequency range up to $4 \mathrm{~Hz}$. These waves are highest in amplitude and slowest. They are seen normally in adults during sleep and in babies. These are typically most prominent frontally in adults and posteriorly in babies. 
Theta is the waves having a frequency range from $4 \mathrm{~Hz}$ to $7 \mathrm{~Hz}$.[2] Theta waves are generally seen in young people. They may be seen in drowsiness or arousal in older children and adults or can also be in meditation .Excess theta for age represents abnormal activity.

Alpha is the waves having a frequency range from $8 \mathrm{~Hz}$ to $12 \mathrm{~Hz}$. This activity is seen in the posterior regions of the head on both sides, having a higher amplitude on the dominant side.

Beta is the waves having a frequency range from $12 \mathrm{~Hz}$ to about $30 \mathrm{~Hz}$. They are usually seen on both sides in symmetrical distribution and is most evident frontally. Beta activity is closely associated to busy or anxious thinking and active concentration.. It is the dominant wave in patients who are alert or anxious or who have their eyes open.

Gamma is the waves having frequency range $30-100 \mathrm{~Hz}$. Gamma rhythms are represent short term memory toning of recognizable objects,sound and tactile sensations.

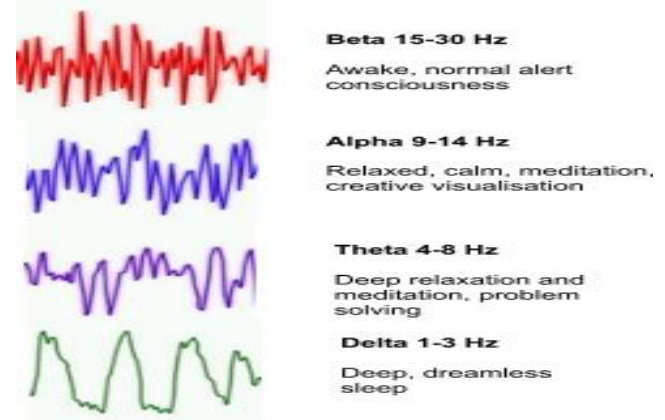

Figure 3. EEG Waves

\section{10-20 System of Electrode Placement-}

The 10-20 System of Electrode Placement is the method which is used to describe the location of electrodes which are implanted in the scalp. The numbers 10 and 20 shows that the distance between adjacent electrodes are $10 \%$ or $20 \%$ of total front or right left distance. Scalp electrodes are used to record the electroencephalogram (EEG) with the help of a machine called an electroencephalograph. The EEG is the record of activity of the brain. This record is the result of the activity of neurons in the brain. This pattern changes with the level of a person's provocation - if a person is relaxed, then the EEG has slow waves and if excited, the EEG has many fast waves. The EEG is used to record brain activity for many purposes including sleep research and to help in the diagnosis of brain disorders like epilepsy.

The 10-20 system is based on the connection between the location of an electrode and the primary area of cerebral cortex. Each point on the left indicates an electrode position. Each site has an alphabet which identifies the lobe and a number or another letter which identifies the hemisphere location. The alphabets F, T, C, P, and $\mathrm{O}$ abbreviated as Frontal, Temporal, Central, Parietal and Occipital. It is important to note that there is no "central lobe", but it is just used for only identification purposes. Even numbers $(2,4,6,8)$ refers the right hemisphere and odd numbers $(1,3,5,7)$ refers the left hemisphere[8]. The $\mathrm{z}$ refers an electrode which is placed on the median. Also note that the smaller is the number, closer is the position to median. 


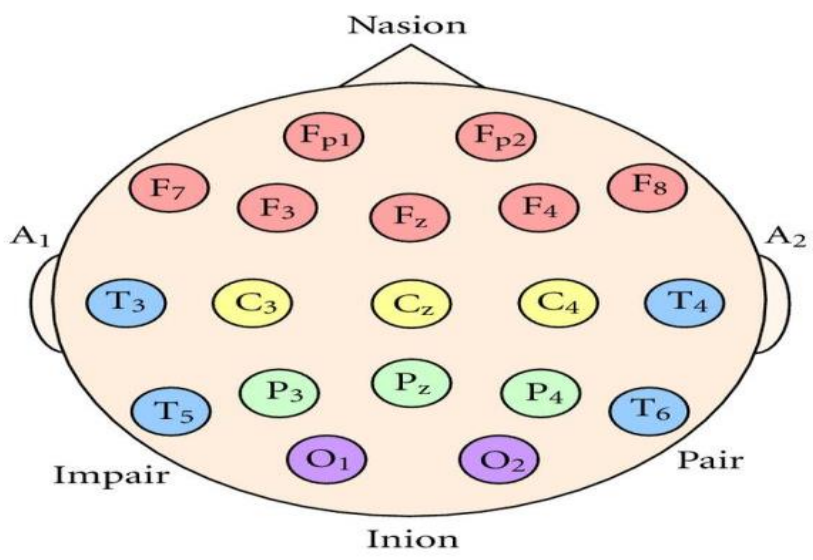

Figure 4. Electrode Placement (The "10" and "20" refer to the $10 \%$ or $20 \%$ Interelectrode Distance)

\section{Mode of Operation}

Here we use Polysomnogrsphy Hardware System RMS -32 with 32 channels is used for simultaneous data acquisition. RMS-32 is a very useful hardware system which is used to acquire the signal by surface electrodes. The software used is SUPERSEC which consists of two software Acquire and Analysis. There are various settings of montage available in the software:

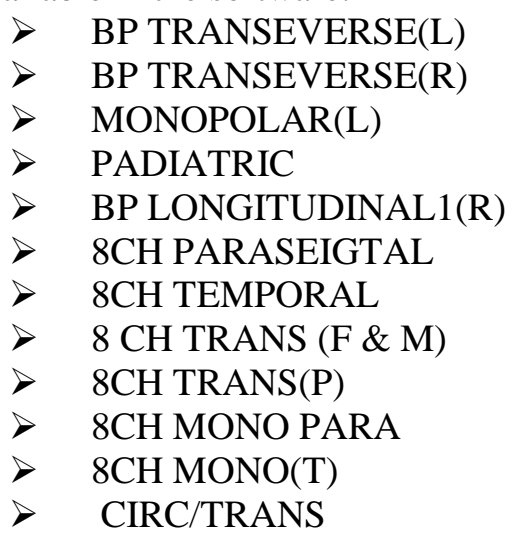

EEG signal of 15 healthy persons under three conditions viz.. While talking on GSM phone $2 \mathrm{G}$ and $3 \mathrm{G}$ is acquired. After the acquisition of EEG signal, different DSP techniques like digital filter processing, fast Fourier transform, autocorrelation, cross correlation is used to process the EEG signal.Acquire is used to record the signals and BP LONGITUDINAL 1(R) configuration for montage is set for conducting this study. [9]The setting of montage used in this study for the signal acquisition has been shown in Figure no 5. This configuration has 16 electrodes. Analysis Software is used to analyze the signals so acquied 


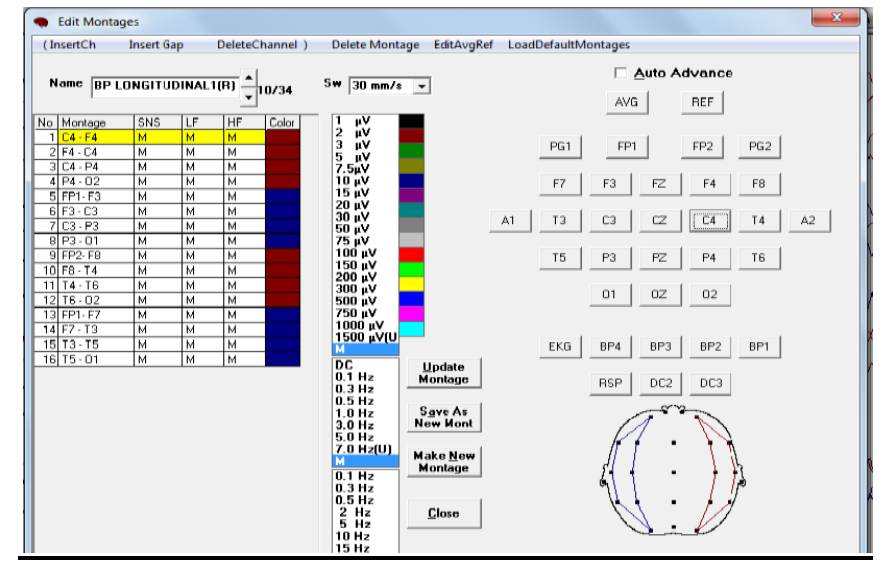

Figure 5. Montage Setting for EEG Signal Acquisition [28]

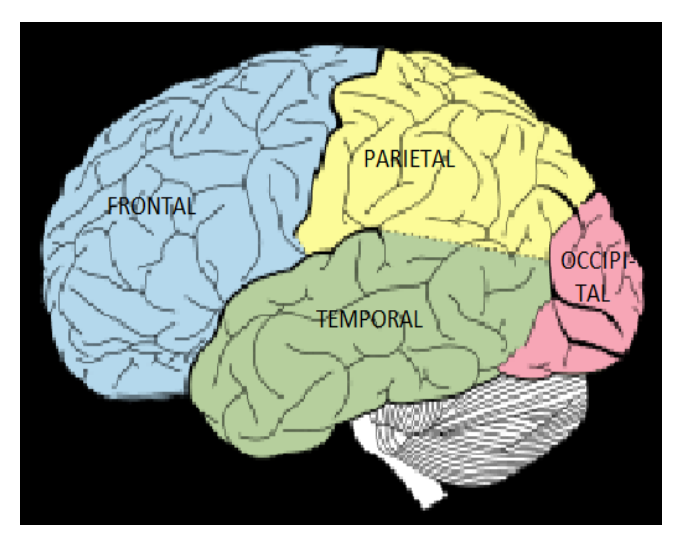

Figure 6. Regions of Brain [29]

The channels with even numbers correspond to right hemisphere of brain and the one with odd numbers correspond to left hemisphere of brain. The letters F, P, T and O correspond to Frontal, parietal, Temporal and Occipital region of brain respectively. The regions of brain corresponding to channels on EEG montage are shown in Figure 6.

\section{Analysis Of EEG Signal}

\subsection{Selection OF Channels for Analysis}

BP LONGITUDINAL 1(R) setting of Montage has 16 channels. Out of 16 channels, the most significant 8 , which are near the ear regions, have been chosen for analyses which are:

1. FP1-F7

2. $\mathrm{F} 7-\mathrm{T} 3$

3. T3-T5

4. T5-O1

5. FP2-F8

6. F8-T4

7. T4-T6

8. T6-O2

The first four channels are in the scalp region near the left ear and last four are near right ear. As during conversation, phone is held at left ear by the subject, the most significant eight channels (FP1-F7, F7-T3, T3-T5, T5-O1, F8-T4, T4-T6, FP2-Fp8, T6$\mathrm{O} 2)$, which are in the immediate vicinity of left ear are selected for the further analyses. 


\subsection{Sample Rate}

The "Sample Rate" value indicates how many samples the RMS 32 should acquire per second on each channel during data acquisition. The default Sample Rate is $121 \mathrm{~Hz}$, but can be changed by clicking on the pull-down menu[8]. Depending on the nature of the data being acquired, the "best" choice for Sample Rate will vary.

\subsection{Acquisition Length}

There is no limit on how long a signal can be recorded. Clinical application require a recording time of 40 to 45 minutes. Even over night recording is also desired sometimes while studying sleep stages[9]. In this study duration of 10 minutes has been used as recording period.The time varying EEG signal and its power spectral density plot calculated by applying Fast Fourier transform on continuous time varying EEG signal as shown in Figure 7 and Figure 8.

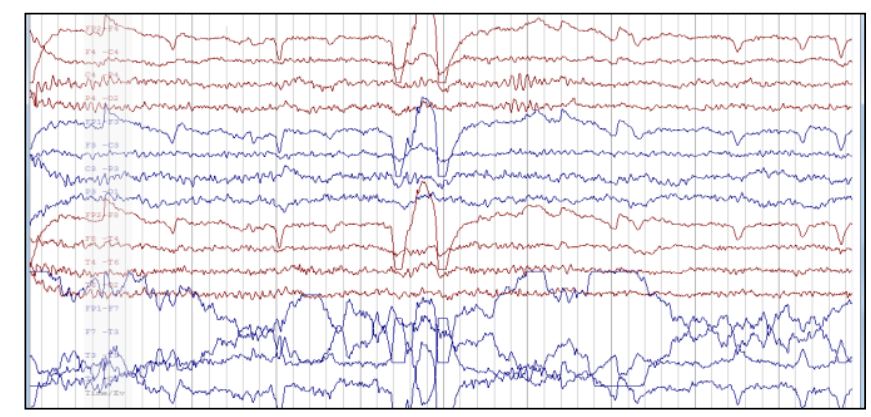

Figure 7. Analog EEG Signal [28]

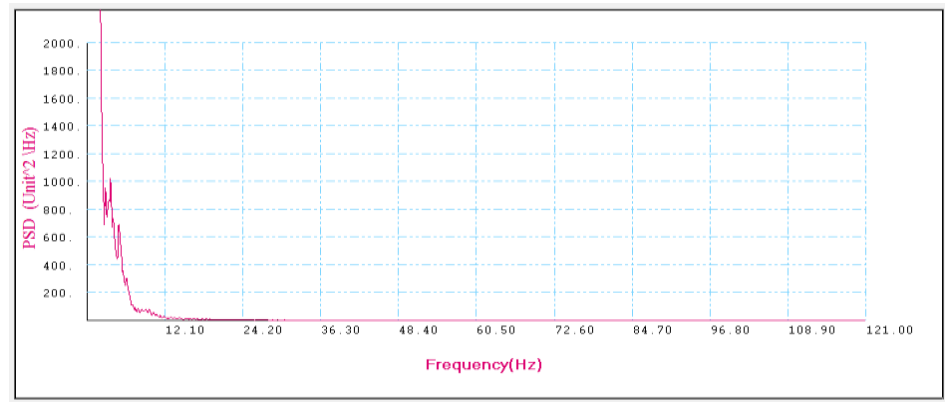

Figure 8. FFT Plot of EEG Signal [28]

The values of Power Spectral Density are exported to MS Excel and the data so obtained is analyzed by applying various mathematical tools such as Min, Max, Average, Auto Correlation and Cross Correlation to evaluate the effect of different communication technologies on human brain activity.

\section{Results Analysis}

The signals obtained under three different scenarios are analyzed with digital signal processing techniques such as Autocorrelation and Power Spectral Density analyses. Table 1 shows the power spectral density (PSD) in two modes of communication including wireless (GSM 2G and GSM 3G ) and other state being IDLE (with no radiation in vicinity of subject) for 8 channels (FP1-F7, F7-T3, T3-T5, T5-O1,FP2-FP8,T6-02,F8T4and F8-T4) of EEG montage for all the subjects. The average PSD values of all the subjects show that GSM 2 G operated phone has highest effect on brain activity followed by GSM $3 \mathrm{G}$ and brain activity is found to be least when subject is sitting IDLE. 
Table 1. Average Psd Value of Different Subjects

\begin{tabular}{|c|c|c|c|}
\hline Channel & GSM 3G & GSM 2G & IDLE \\
\hline F7-T3 & 1503.361 & 22601.23 & 906.7354 \\
\hline F8-T4 & 3536.639 & 20446.05 & 2336.99 \\
\hline T3-T5 & 2832.49 & 3815.121 & 1612.059 \\
\hline T4-T6 & 10363.55 & 13584.31 & 9391.605 \\
\hline FP1-FP7 & 12947.24 & 22601.22 & 10987.97 \\
\hline FP2-FP8 & 15140.17 & 20446.05 & 13509.75 \\
\hline T5-O1 & 7301 & 21306.04 & 5161.344 \\
\hline T6-02 & 27630 & 108387.4 & 21279.31 \\
\hline
\end{tabular}

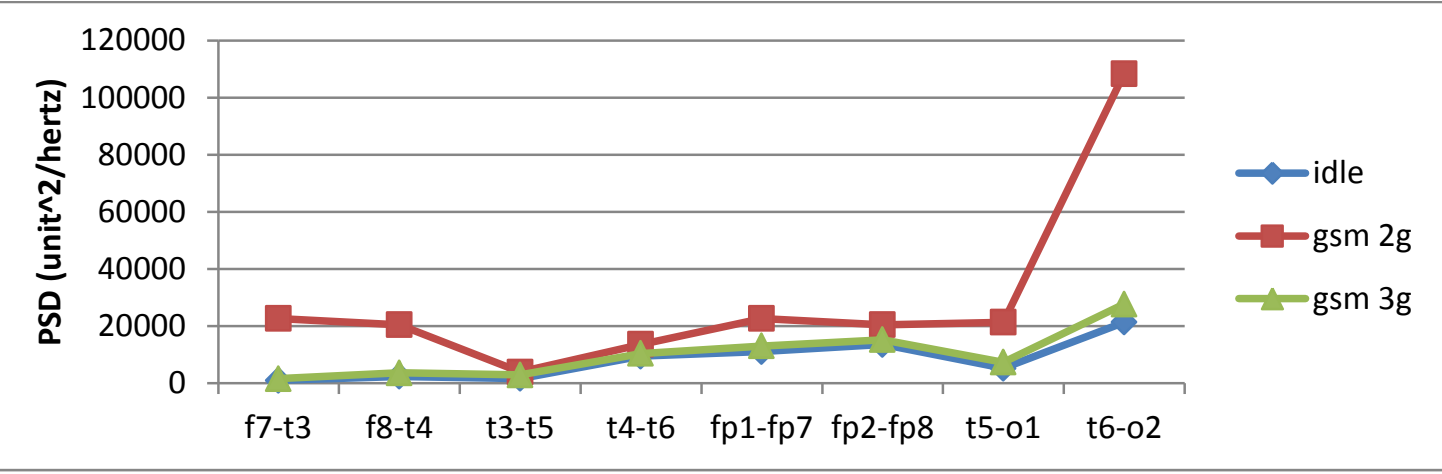

Figure 9. Bar Plot of Average PSD Values of all Subjects

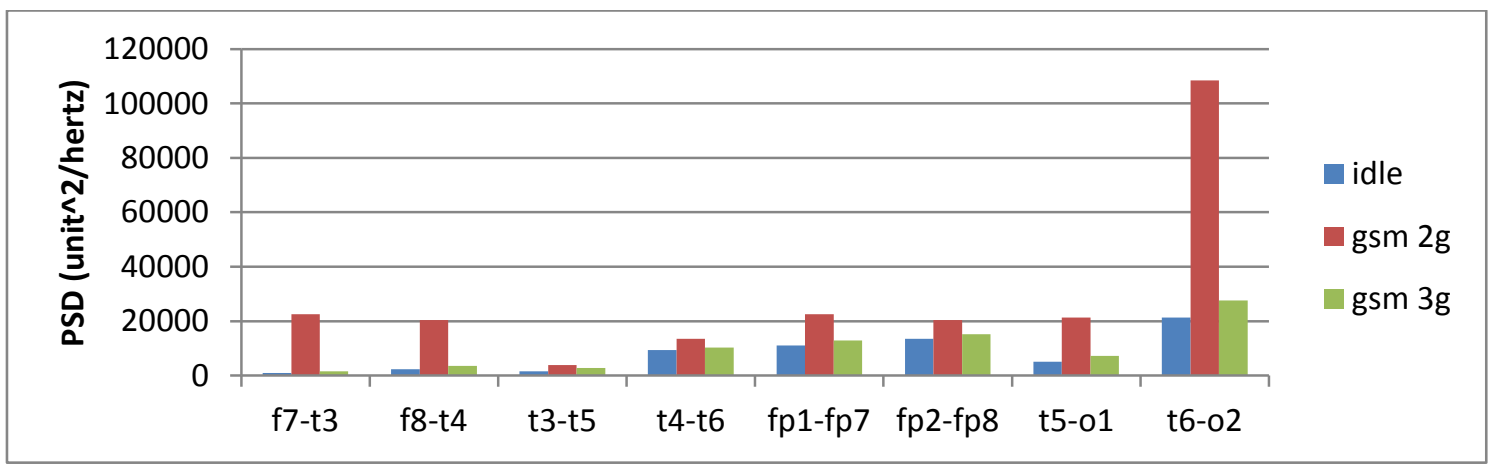

Figure 10. Plot of Average PSD Values of all Subjects

\section{Conclusion}

The signals obtained under three different scenarios are analyzed with digital signal processing techniques such as Autocorrelation and Power Spectral Density analyses. Table 1 shows the power spectral density (PSD) in two modes of communication including wireless (GSM 2G and GSM 3G) and other state being IDLE (with no radiation in vicinity of subject) for 8 channels (FP1-F7, F7-T3, T3-T5, T5-O1,FP2-FP8,T6-02,F8T4and F8-T4) of EEG montage for all the subjects. The average PSD values of all the subjects show that GSM 2G operated phone has highest effect on brain activity followed by GSM $3 \mathrm{G}$ and brain activity is found to be least when subject is sitting IDLE.

\section{References}

[1] A. Tyagi, M. Duhan and D. Bhatia, "Effect Of Mobile Phone Radiation On Brain Activity GSM Vs CDMA", IJSTM, vol. 2, iss. 2, (2011). 
[2] E. Niedermeyer and F. L. da Silva. "Electroencephalography: Basic Principles", Clinical Applications and related fields. Lippincot Williams and Wikkins, (2004).

[3] D. A. A. Mat, "The Effect of Handset and Earphone on Reducing Electromagnetic Radiation from Mobile Phone towards Human Head", IEEE, (2009).

[4] A. Y. Simba, S. Watanabe, T. Hikage and T. Nozima, "A Review of Mobile Phone Usage in Enclosed Areas and RF safety Guidelines”,IEEE AFRICON, (2009).

[5] C. Kargel, "Infrared Thermal Imaging To Measure Local Temperature Rises Control By Hand Held Mobile Phones", IEEE Transaction On Instrumentation And Measurement, (2005).

[6] "GSM world statistics", GSM Association, (2010).

[7] "GSM Technical Data", Cellular.co.za, http ://www.cellular.co.za/gsmtechdata.

[8] A. Y. Simba, S. Watanabe, T. Hikage and T. Nozima, "A Review of Mobile Phone Usage in Enclosed Areas and RF safety Guidelines", IEEE AFRICON, (2009).

[9] C. Kargel, "Infrared Thermal Imaging To Measure Local Temperature Rises Control By Hand Held Mobile Phones", IEEE Transaction On Instrumentation And Measurement, (2005).

[10] J. D. Kraus, "Electromagnetic International Edition", 4 ${ }^{\text {th }}$ Edition, New York:Mc Graw Hill, (1992).

[11] D. W. Ricker, EEG signal processing, Springer, (2003).

[12] A. F. Molisch, Wireless communication( $2^{\text {nd }}$ Edition $)$, John Wiley and Sons, (2011).

[13] J. B. Ochoa, "EEG Signal Classification For Brain Computer Interface Applications", (2002).

[14] midpdf.com/result-standard-international-10-20-electrode-placement-pdf.html, http://www.ebme.co.uk/, en.wikipedia.org/wiki/Electromagnetic Radiation.

[15] R. F. Safe, "What is RF Radiation",http://www.rsafe.com, "Two billion gsm customers worldwide ", 3G America, http://www.prnewswire.com/cgi-bin/stories.pl.2010.

[16] "Better Health Channel", Mobile Phones and You Health, http://www.betterhealth.vic.gov.au/bhcv2/articles.nsf/pages/mobile_phones_and_youhealth?,

"Electromagnetic Frequencies", Thermal Effects of Electromagnetic Radiation, http://cellphonesafety.wordpress.com/2006/07/31, (2008).

[17] R. F. Safe, "What is RF radiation?", http://www.rsafe.com/research/rf/radiation/what_is_rf/intro.html, (2008).

[18] “Thermal Radiation”, http://en.wikipedia.org/wiki Thermal Radiation, (2008).

[19] Mobile Phone Stimulations With Human Brain And Human Hand Models, http://www.cst.com/content/applications/article/mobile

[20] en.wikipedia.org/wiki/GSM

[21] en.wikipedia.org/wiki/CDMA

[22] http://en.wikipedia.org/wiki/cross-correlation

[23] http://en.wikipedia.org/wiki/auto-correlation

[24] http://en.wikipedia.org/wiki/action potential

[25] RMS :Superspec version 4.2.6,Copyright 2007-2008 Recorder and Medicare System(p)Ltd

[26] http://en.wikipedia.org/wiki/brain

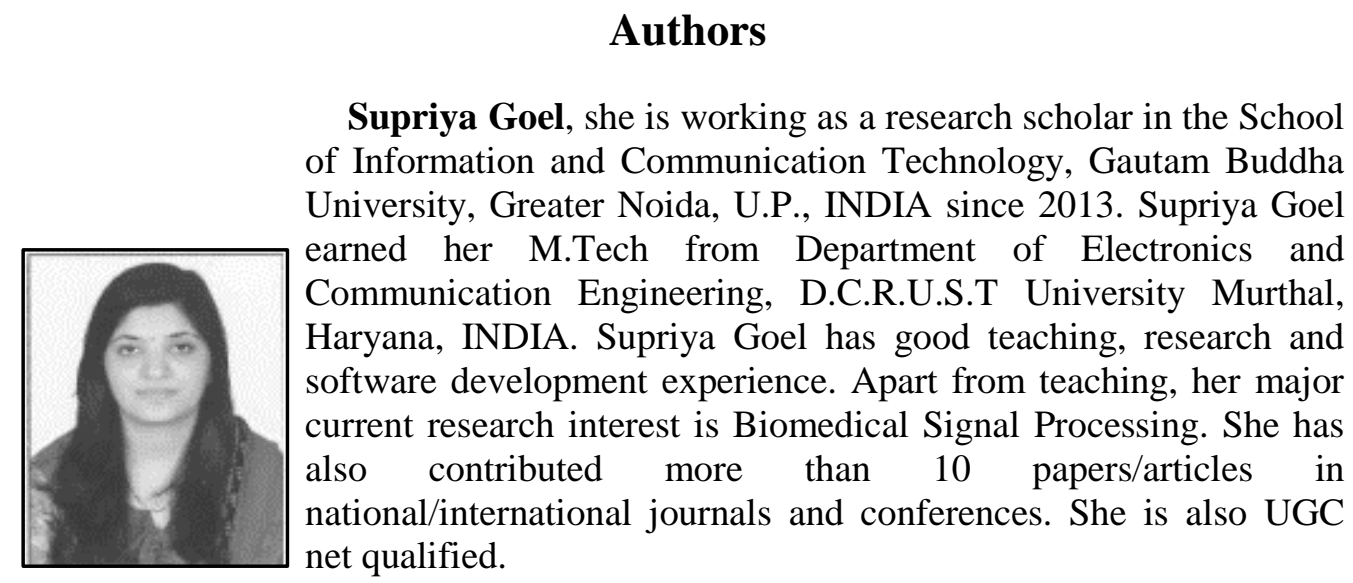

